\title{
A Complex Neural Network Algorithm for Computing the Largest Real Part Eigenvalue and the corresponding Eigenvector of a Real Matrix
}

\author{
HANG TAN ${ }^{1, a}$, XUESONG LIANG ${ }^{1, b}$ and LIPING WAN ${ }^{1, c}$ \\ ${ }^{1}$ College of Physics and Engineering, Chengdu Normal University, Chengdu 611130, China \\ ahangtancdnu@163.com, bliang_xuesong@sohu.com, clpwan2013@126.com
}

\begin{abstract}
Keywords: Complex neural network; real matrix; largest real part; eigenvalue; eigenvector
Abstract. In this study, we propose a novel complex neural network algorithm, which extends the neural network based approaches that can asymptotically compute the largest or smallest eigenvalues and the corresponding eigenvectors of real symmetric matrices, to the case of directly calculating the largest real part eigenvalue and the corresponding eigenvector of a real matrix. The proposed neural network algorithm is described by a group of complex differential equations, which is deduced from the classical neural network model. The proposed algorithm is a class of continuous time recurrent neural network (RNN), it has parallel processing ability in an asynchronous manner and could achieve high computing capability. This paper provides a rigorous mathematical proof for its convergence in the case of real matrices for a more clear understanding of network dynamic behaviors relating to the computation of eigenvector and eigenvalue. The proposed approach has obvious virtues such as fast convergence speed and non-sensitivity to initial value. Numerical examples showed that the proposed algorithm has good performance.
\end{abstract}

\section{Introduction}

Using the neural network technology to extract the eigenvector corresponding to the modulus maximum eigenvalue of a real symmetric matrix was first presented about 30 years ago [1], then motivated broad interests from engineering and theoretical researches [2-12]. In 1995, Luo et al. [5, 6] proposed a very classical neural network algorithm for extracting not only modulus maximum eigenvalue but also modulus minimum eigenvalue and their corresponding eigenvectors of real symmetric matrices, but the convergence proof of this algorithm not be presented until 2004 by Zhang et al. [7]. In the recently years, some adaptive generalized eigen-pairs extraction algorithms have been presented by some authors $[11,12]$.

A number of years ago, Liu et al. [3] proposed a simpler neural network algorithm for extracting the eigenvector corresponding to the modulus maximum eigenvalue of a real symmetric matrix, but it will also diverge as the same as in the publication [1] when it is used to extract the eigenvector corresponding to the modulus minimum eigenvalue of a real symmetric matrix. Then Liu et al. [4] presented a subtle method to extract the imaginary part of the eigenvalue from the maximum imaginary part and the real part of the eigenvalue from the maximum real part of a general real matrix. Unfortunately, they couldn't obtain the corresponding eigenvector, which is sometimes vital for some practical engineering problems. In the present paper, we try to not only extract the largest real part eigenvalue but also extract the corresponding eigenvector of any real matrix in the complex domain.

The rest of this paper is organized as follows: In section 2, we proposed the novel complex domain neural network model of this paper. Some numerical examples given in section 3 and we summarized this paper in the last section.

\section{The Proposed Complex Neural Network Model}

The classical neural network algorithm for computing the eigenvector corresponding to the modulus maximum eigenvalue or modulus minimum eigenvalue can be illustrated as follows $[5,6]$ 


$$
\frac{d v(t)}{d t}=v(t)^{T} v(t) A v(t)-v(t)^{T} A v(t) v(t),
$$

where $v(t) \in R^{n}$ are the $n$ dimension real column vectors that denote the states of neurons. However, the above classical neural network algorithm can only be used to solve the eigen-pair problems of real symmetric matrices. We could not directly adopt it to compute the eigen-pair problems of general real matrices. Now we substitute matrix $A$ in Eq. 1 by the following $A^{\prime}$ :

$$
A^{\prime}=\left(\begin{array}{cc}
A & 0 \\
0 & A
\end{array}\right)
$$

where $A$ is a general real matrix, $v(t) \in R^{2 n}$, let

$$
v(t)=\left[\begin{array}{l}
x(t) \\
y(t)
\end{array}\right],
$$

one can get $v(t)^{T}=\left[x(t)^{T}, y(t)^{T}\right]$, so Eq. 1 can be converted into the following forms

$$
\begin{aligned}
& \frac{d x(t)}{d t}=\sum_{j=1}^{n}\left[x_{j}^{2}+y_{j}^{2}\right] A x(t)-\left[x(t)^{T} A x(t)+y(t)^{T} A y(t)\right] x(t), \\
& \frac{d y(t)}{d t}=\sum_{j=1}^{n}\left[x_{j}^{2}+y_{j}^{2}\right] A y(t)-\left[x(t)^{T} A x(t)+y(t)^{T} A x(t)\right] y(t) .
\end{aligned}
$$

Add the first equation with the second equation times $\mathrm{i}$ of Eq. 2, where $\mathrm{i}$ is an imaginary unit. Assume that $z(t)=x(t)+y(t) \mathrm{i}$, one gets

$$
\frac{d z(t)}{d t}=z(t)^{T} \bar{z} A z(t)-\left[x(t)^{T} A x(t)+y(t)^{T} A y(t)\right] z(t) .
$$

Consider that $x(t)^{T} A x(t)+y(t)^{T} A y(t)=\frac{z(t)^{T} A \overline{z(t)}^{2}+\bar{z}^{T}{ }^{T} A z(t)}{2}$, Eq. 3 becomes

$$
\frac{d z(t)}{d t}=z(t)^{T} \overline{z(t)} A z(t)-\frac{\overline{z(t)}^{T} A z(t)+z(t)^{T} A \overline{z(t)}}{2} z(t),
$$

which is the complex neural network model in our study, where $z(t) \in C^{n}$. The algorithm can be used to extract the eigenvector and the corresponding eigenvalue with largest real part of a general real matrix.

If $A \in R^{n \times n}$ is a real matrix, and let $\lambda_{1} \geq \lambda_{2} \geq \cdots \geq \lambda_{n}$ are $n$ eigenvalues of $A$, then the corresponding normal basic complex eigenvectors are $S_{1}, S_{2}, \cdots, S_{n}$. Let $z(t)=x(t)+\mathrm{i} y(t)$, one gets

$$
z(t)=\sum_{k=1}^{n} z_{k}(t) S_{k}=\sum_{k=1}^{n}\left[x_{k}(t)+\mathrm{i} y_{k}(t)\right] S_{k},
$$

where $z_{k}(t)=x_{k}(t)+\mathrm{i} y_{k}(t)$ denote the projection of $z(t)$ in the direction along with $S_{k}$.

Theorem 1. Let $|z(t)|^{2}=\overline{z(t)}^{T} z(t)=z(t)^{T} \overline{z(t)}$, and for any non-zero initial value $z(0) \in C^{n}$, the solution of Eq. 4 satisfying $|z(t)|^{2}=|z(0)|^{2}$.

Proof: Due to $\frac{d|z|^{2}}{d t}=\frac{d\left(\bar{z}^{T} z\right)}{d t}=\bar{z}^{T} \dot{z}+z^{T} \dot{\bar{z}}$, and 


$$
\begin{aligned}
& \bar{z}^{T} \dot{z}+z^{T} \dot{\bar{z}}=\bar{z}^{T}\left\{z^{T} \bar{z} A z-\frac{\bar{z}^{T} A z+z^{T} A \bar{z}}{2} z\right\}+z^{T}\left\{\bar{z}^{T} z A \bar{z}-\frac{z^{T} A \bar{z}+\bar{z}^{T} A z}{2} \bar{z}\right\} \\
& =\left\{\bar{z}^{T} z^{T} \bar{z} A z-\bar{z}^{T} \frac{\bar{z}^{T} A z}{2} z-z^{T} \frac{\bar{z}^{T} A z}{2} \bar{z}\right\}+\left\{z^{T} \bar{z}^{T} z A \bar{z}-\bar{z}^{T} \frac{z^{T} A \bar{z}}{2} z-z^{T} \frac{z^{T} A \bar{z}}{2} \bar{z}\right\} \\
& =0+0=0 .
\end{aligned}
$$

One can get $\frac{d\left(\left.z(t)\right|^{2}\right)}{d t}=0$, i.e. $|z(t)|^{2}$ is a constant, in other words, so one can obtain $|z(t)|^{2}=z(t)^{T} \overline{z(t)}=z(0)^{T} \overline{z(0)}=|z(0)|^{2}$, thus complete proof.

Theorem 2. If the complex vector $z(t)$ is the solution of Eq. 4 and $z_{k}(t)=x_{k}(t)+\mathrm{i} y_{k}(t)$ denotes the projection of $z(t)$ along with the direction $S_{k}$, then $|z(t)|^{2}$ can be read as

$$
|z(t)|^{2}=\frac{\sum_{k=1}^{n} \exp \left[2 z(0)^{T} \overline{z(0)} \lambda_{k}^{R} t\right] \times\left|z_{k}(0)\right|^{2}}{1+2 \sum_{j=1}^{n}\left|z_{j}(0)\right|^{2} \lambda_{j}^{R} \int_{0}^{t} \exp \left[2 z(0)^{T} \overline{z(0)} \lambda_{j}^{R} \tau\right] d \tau} .
$$

Proof: We first need to prove that the projection of $z(t)$ onto $S_{k}$ can be represented as:

$$
\left|z_{k}(t)\right|^{2}=\frac{\exp \left[2 z(0)^{T} \overline{z(0)} \lambda_{k}^{R} t\right] \times\left|z_{k}(0)\right|^{2}}{1+2 \sum_{j=1}^{n}\left|z_{j}(0)\right|^{2} \lambda_{j}^{R} \int_{0}^{t} \exp \left[2 z(0)^{T} \overline{z(0)} \lambda_{j}^{R} \tau\right] d \tau} .
$$

Assume that all eigenvalues of $A$ can be denoted as $\lambda_{k}^{R}+\mathrm{i} \lambda_{k}^{I}, k=1, \cdots, n$, where $\lambda_{k}^{R}$ and $\lambda_{k}^{I}$ are the real part and the imaginary part of eigenvalue $\lambda_{k}$, respectively. Let $z(t)=x(t)+\mathrm{i} y(t)$, and assume that the solution $z(t)$ of Eq. 4 can be represent as follows:

$$
z(t)=\sum_{k=1}^{n}\left[x_{k}(t)+\mathrm{i} y_{k}(t)\right] S_{k} \text {. }
$$

Substituting Eq. 8 into Eq. 4, one gets the following form when $t \rightarrow \infty$ :

$$
\sum_{k=1}^{n}\left[\frac{d x_{k}(t)}{d t}+\mathrm{i} \frac{d y_{k}(t)}{d t}\right] S_{k}=\sum_{j=1}^{n}\left|z_{j}\right|^{2} \sum_{k=1}^{n} \lambda_{k}\left(x_{k}+\mathrm{i} y_{k}\right) S_{k}-\frac{1}{2}\left\{\sum_{j=1}^{n}\left[\lambda_{j}+\overline{\lambda_{j}}\right]\left|z_{j}\right|^{2}\right\} \sum_{k=1}^{n}\left(x_{k}+\mathrm{i} y_{k}\right) S_{k} \text {. }
$$

Along with $S_{k}$, the following equation can be obtained:

$$
\frac{d x_{k}(t)}{d t}+\mathrm{i} \frac{d y_{k}(t)}{d t}=\sum_{j=1}^{n}\left|z_{j}\right|^{2} \lambda_{k}\left(x_{k}+\mathrm{i} y_{k}\right)-\frac{1}{2}\left\{\sum_{j=1}^{n}\left[\lambda_{j}+\overline{\lambda_{j}}\right]\left|z_{j}\right|^{2}\right\}\left(x_{k}+\mathrm{i} y_{k}\right) .
$$

Let $\lambda_{j}=\lambda_{j}^{R}+\mathrm{i} \lambda_{j}^{I}, \bar{\lambda}_{j}=\lambda_{j}^{R}-\mathrm{i} \lambda_{j}^{I}, \lambda_{k}=\lambda_{k}^{R}+\mathrm{i} \lambda_{k}^{I}$, insert them into Eq. 10. After separating the real part and the imaginary part, we have:

$$
\begin{aligned}
& \frac{d x_{k}(t)}{d t}=\sum_{j=1}^{n}\left|z_{j}\right|^{2}\left(\lambda_{k}^{R} x_{k}-\lambda_{k}^{I} y_{k}\right)-\sum_{j=1}^{n}\left|z_{j}\right|^{2} \lambda_{j}^{R} x_{k}, \\
& \frac{d y_{k}(t)}{d t}=\sum_{j=1}^{n}\left|z_{j}\right|^{2}\left(\lambda_{k}^{R} y_{k}+\lambda_{k}^{I} x_{k}\right)-\sum_{j=1}^{n}\left|z_{j}\right|^{2} \lambda_{j}^{R} y_{k} .
\end{aligned}
$$

As $\frac{d\left|z_{k}\right|^{2}}{d t}=\frac{d\left(\overline{z_{k}} z_{k}\right)}{d t}=2 x_{k} \frac{d x_{k}}{d t}+2 y_{k} \frac{d y_{k}}{d t}$, based on Eq. 11, we can obtain the following equation:

$$
\frac{d\left|z_{k}(t)\right|^{2}}{d t}=2 \sum_{j=1}^{n}\left|z_{j}(t)\right|^{2} \lambda_{k}^{R}\left|z_{k}(t)\right|^{2}-2 \sum_{j=1}^{n}\left|z_{j}(t)\right|^{2} \lambda_{j}^{R}\left|z_{k}(t)\right|^{2}=2 \sum_{j=1}^{n}\left|z_{j}(t)\right|^{2}\left|z_{k}\right|^{2}\left(\lambda_{k}^{R}-\lambda_{j}^{R}\right) \text {. }
$$

Based on the theorem 1, Eq. 12 can be converted into the following form: 


$$
\begin{aligned}
& \frac{d\left|z_{k}(t)\right|^{2}}{d t}=2 \sum_{j=1}^{n}\left|z_{j}(t)\right|^{2} \lambda_{k}^{R}\left|z_{k}(t)\right|^{2}-2 \sum_{j=1}^{n} \lambda_{j}^{R}\left|z_{j}(t)\right|^{2}\left|z_{k}(t)\right|^{2} \\
& =2 z(0)^{T} \overline{z(0)}\left|z_{k}(t)\right|^{2} \lambda_{k}^{R}-2 \sum_{j=1}^{n}\left|z_{j}(t)\right|^{2} \lambda_{j}^{R}\left|z_{k}(t)\right|^{2} . \\
& \text { If }\left|z_{k}(t)\right|^{2} \neq 0,\left|z_{r}(t)\right|^{2} \neq 0 \text {, then } \\
& \frac{1}{\left|z_{k}(t)\right|^{2}} \frac{d}{d t}\left|z_{k}(t)\right|^{2}-\frac{1}{\left|z_{r}(t)\right|^{2}} \frac{d}{d t}\left|z_{r}(t)\right|^{2}=2 z(0)^{T} \overline{z(0)}\left(\lambda_{k}^{R}-\lambda_{r}^{R}\right),
\end{aligned}
$$

Therefore

$\frac{d}{d t}\left\{\ln \frac{\left|z_{k}(t)\right|^{2}}{\left|z_{r}\right|^{2}}\right\}=2 z(0)^{T} \overline{z(0)}\left(\lambda_{k}^{R}-\lambda_{r}^{R}\right)$,

i.e. :

$\frac{\left|z_{k}\right|^{2}}{\left|z_{r}\right|^{2}}=\frac{\left|z_{k}(0)\right|}{\left|z_{r}(0)\right|^{2}} \exp \left[2 z(0)^{T} \overline{z(0)}\left(\lambda_{k}^{R}-\lambda_{r}^{R}\right) t\right]$.

If $\left|z_{k}(0)\right|^{2} \neq 0$, therefore $z_{k}(t) \neq 0$, then Eq. 13 can be directly written as follows:

$\frac{1}{\left|z_{k}\right|^{4}} \frac{d}{d t}\left|z_{k}\right|^{2}=2 z(0)^{T} \overline{z(0)} \lambda_{k}^{R} \frac{1}{\left|z_{k}\right|^{2}}-2 \sum_{j=1}^{n} \frac{\left|z_{j}\right|^{2}}{\left|z_{k}\right|^{2}} \lambda_{j}^{R}$,

i.e.

$$
\frac{d}{d t} \frac{1}{\left|z_{k}(t)\right|^{2}}+2 z(0)^{T} \overline{z(0)} \lambda_{k}^{R} \frac{1}{\left|z_{k}(t)\right|^{2}}=2 \sum_{j=1}^{n} \frac{\left|z_{j}(t)\right|^{2}}{\left|z_{k}(t)\right|^{2}} \lambda_{j}^{R} .
$$

Substituting Eq.15 into Eq. 17, one gets

$$
\frac{d}{d t} \frac{\exp \left[2 z(0)^{T} \overline{z(0)} \lambda_{k}^{R} t\right]}{\left|z_{k}(t)\right|^{2}}=2 \sum_{j=1}^{n} \frac{\left|z_{j}(0)\right|^{2}}{\left|z_{k}(0)\right|^{2}} \lambda_{j}^{R} \exp \left[2 z(0)^{T} \overline{z(0)} \lambda_{j}^{R} t\right],
$$

The integral on both sides of Eq. 18 from 0 to $t$ reads

$$
\frac{\exp \left[2 z(0)^{T} \overline{z(0)} \lambda_{k}^{R} t\right]}{\left|z_{k}(t)\right|^{2}}-\frac{1}{\left|z_{k}(0)\right|^{2}}=2 \sum_{j=1}^{n} \frac{\left|z_{j}(0)\right|^{2}}{\left|z_{k}(0)\right|^{2}} \lambda_{j}^{R} \int_{0}^{t} \exp \left[2 z(0)^{T} \overline{z(0)} \lambda_{j}^{R} \tau\right] d \tau \text {. }
$$

Direct calculation reads

$$
\left|z_{k}(t)\right|^{2}=\frac{\exp \left[2 z(0)^{T} \overline{z(0)} \lambda_{k}^{R} t\right] \times\left|z_{k}(0)\right|^{2}}{1+2 \sum_{j=1}^{n}\left|z_{j}(0)\right|^{2} \lambda_{j}^{R} \int_{0}^{t} \exp \left[2 z(0)^{T} \overline{z(0)} \lambda_{j}^{R} \tau\right] d \tau},
$$

Therefore

$$
|z(t)|^{2}=\frac{\sum_{k=1}^{n} \exp \left[2 z(0)^{T} \overline{z(0)} \lambda_{k}^{R} t\right] \times\left|z_{k}(0)\right|^{2}}{1+2 \sum_{j=1}^{n}\left|z_{j}(0)\right|^{2} \lambda_{j}^{R} \int_{0}^{t} \exp \left[2 z(0)^{T} \overline{z(0)} \lambda_{j}^{R} \tau\right] d \tau} .
$$

Theorem 3: Solve Eq. 4, after convergence, and let $v=\lim _{t \rightarrow \infty} z(t)$, then $v$ will converge to an eigenvector of the real symmetric matrix $A$, which corresponds to the maximum real part eigenvalue that can be denoted as $\lambda=\frac{\overline{z(t)}^{T} A z(t)+z(t)^{T} A \overline{z(t)}}{2 z(t)^{T} \overline{z(t)}}$. 
Proof: According to the equilibrium point of the Eq. 4 one gets $A z(t)=\frac{\overline{z(t)}^{T} A z(t)+z(t)^{T} A \overline{z(t)}}{2 z(t)^{T} \overline{z(t)}} z(t)$. If we consider $z(t)$ as an eigenvector of a real matrix $A$, then the corresponding eigenvalue can be read as $\lambda=\frac{\overline{z(t)}^{T} A z(t)+z(t)^{T} A \overline{z(t)}}{2 z(t)^{T} z(t)}$. Now we need to prove that $z(t)$ will converge to the eigenvector corresponding to the maximum real part eigenvalue of $A$. Based on the theorem 2, we should prove that $z(t)$ is indeed the linear combination of those eigenvectors corresponding to all eigenvalues that are identical to $\sigma_{1}$. As the Eq. 7, we denote $z_{i}(t)$ with another form as follows:

$$
\begin{aligned}
& z_{i}(t)=\Delta \cos \left(\theta_{i}\right)+\Delta \sin \left(\theta_{i}\right), \text { where } \\
& \Delta=\sqrt{\frac{\exp \left[2 z(0)^{T} \overline{z(0)} \lambda_{k}^{R} t\right] \times\left|z_{k}(0)\right|^{2}}{1+2 \sum_{j=1}^{n}\left|z_{j}(0)\right|^{2} \lambda_{j}^{R} \int_{0}^{t} \exp \left[2 z(0)^{T} \overline{z(0)} \lambda_{j}^{R} \tau\right] d \tau}},
\end{aligned}
$$

where $\theta_{i}$ denotes the intersection angle between $z_{i}(t)$ and unit vector $S_{i}$. Therefore one get

$$
z(t)=\sum_{i=1}^{n}\left[\Delta \cos \left(\theta_{i}\right)+\Delta \sin \left(\theta_{i}\right) \mathrm{i}\right] S_{i} .
$$

As the analysis of the real part and imaginary part of $z(t)$ are similar, we first consider the analysis of real part. First, simplify the $\Delta$, then the real part becomes:

$$
\text { real part }=\sum_{i=1}^{n} \sqrt{\frac{\exp \left[2 z(0)^{T} \overline{z(0)} \lambda_{i}^{R}(t)\right] \times\left|z_{i}(0)\right|^{2}}{2 \sum_{j=1}^{n}\left|z_{j}(0)\right|^{2} \exp \left[2 z(0)^{T} \overline{z(0)} \lambda_{j}^{R} t\right]}} \cos \left(\theta_{i}\right) S_{i} .
$$

Let $I I=2 z(0)^{T} \overline{z(0)}$, and assume that $z(0)$ is a non-zeros initial value complex vector, so $z(0)=\sum_{i=1}^{n} z_{i}(0) S_{i}$. Definition $l=\min \left\{l \mid 1 \leq l \leq n, z_{l}(0) \neq 0\right\}$, then there does exist a $r \in\{1, \cdots, m\}$ subject that $k_{r-1} \leq l \leq k_{r}$. Therefore the real part of $z(t)$ can be read as:

$$
\text { real part }=\lim _{t \rightarrow \infty} \sum_{i=1}^{n} \sqrt{\frac{\exp \left[I I \lambda_{i}^{R} t\right] \times\left|z_{i}(0)\right|^{2}}{2 \sum_{j=1}^{n}\left|z_{j}(0)\right|^{2} \exp \left[I I \lambda_{j}^{R} t\right]}} \cos \left(\theta_{i}\right) S_{i}=\sum_{i=l}^{k_{r}} \sqrt{\frac{\left|z_{i}(0)\right|^{2}}{2 \sum_{j=l}^{k_{r}}\left|z_{j}(0)\right|^{2}}} \cos \left(\theta_{i}\right) S_{i}
$$

Using the similar method, we have:

$$
\text { imaginary part }=\mathrm{i} \sum_{i=l}^{k_{r}} \sqrt{\frac{\left|z_{i}(0)\right|^{2}}{2 \sum_{j=l}^{k_{r}}\left|z_{j}(0)\right|^{2}}} \sin \left(\theta_{i}\right) S_{i},
$$

Therefore

$$
\lim _{t \rightarrow \infty} z(t)=\left[\sum_{i=l}^{k_{r}} \sqrt{\frac{\left|z_{i}(0)\right|^{2}}{2 \sum_{j=l}^{k_{r}}\left|z_{j}(0)\right|^{2}}} \cos \left(\theta_{i}\right)+\mathrm{i} \sum_{i=l}^{k_{r}} \sqrt{\frac{\left|z_{i}(0)\right|^{2}}{2 \sum_{j=l}^{k_{r}}\left|z_{j}(0)\right|^{2}}} \sin \left(\theta_{i}\right)\right] S_{i} \in V_{\sigma_{r}} .
$$

Therefore, $z(t)$ belongs to the eigen-subspace $V_{\sigma_{r}}$, which should be spanned by all eigenvectors corresponding to the eigenvalues $\sigma_{r}$. Furthermore, if $l=1$, then $z_{l}(0) \neq 0$, i.e., the projection of the initial value $z(0)$ onto the unit eigenvector $S_{1}$ are non-zero. In this case, only $r=1$ can satisfy the condition that $k_{r-1} \leq 1 \leq k_{r}$. Therefore the steady-state solution $z(t) \in V_{\sigma_{1}}$, as before $\sigma_{1}$ denotes the modulus maximum eigenvalue. In practice, as the dimension of $V_{\sigma_{1}}$ is less than $C^{n}$, so the stochastic 
non-zeros initial value isn't always orthogonal to the eigen-subspace $V_{\sigma_{1}}$. Therefore we can always obtain the eigenvector corresponding to the modulus maximum eigenvalue.

\section{Simulation Results}

Example 1. Consider the following $6 \times 6$ real symmetric matrix $A$ :

$$
A=\left(\begin{array}{rrrrrr}
0.7015 & -0.8849 & -0.1655 & -0.9900 & -0.8059 & 0.1416 \\
-0.8849 & 0.0335 & -0.7978 & 0.2970 & -0.2240 & -0.4440 \\
-0.1655 & -0.7978 & -1.7502 & -1.1441 & 0.0937 & -0.6365 \\
-0.9900 & 0.2970 & -1.1441 & 0.9642 & 0.1934 & -0.4340 \\
-0.8059 & -0.2240 & 0.0937 & 0.1934 & -0.7145 & 0.1156 \\
0.1416 & -0.4440 & -0.6365 & -0.4340 & 0.1156 & 2.5260
\end{array}\right) .
$$

We use the following stochastic initial value $z(0)$ for running the complex neural network algorithm Eq. 4 to obtain the largest real part eigenpair of matrix $A$ :

$$
z(0)=\left(\begin{array}{c}
1.6555-1.3320 \mathrm{i} \\
0.3075-2.3299 \mathrm{i} \\
-1.2571-1.4491 \mathrm{i} \\
-0.8655+0.3335 \mathrm{i} \\
-0.1765+0.3914 \mathrm{i} \\
0.7914+0.4517 \mathrm{i}
\end{array}\right)
$$

Directly solving the eigen-pair problems of $A$, we can obtain the eigenvalues of $A$ use matlab function, one gets $\lambda_{1} \sim \lambda_{6}=\{-2.6394,-1.2519,0.0979,0.4253,2.1841,2.9446\}$, and the largest real part eigenvector is:

$$
v_{6}=(-0.3605,0.2748,-0.0275,0.4153,0.0590,-0.7860)^{T} \text {. }
$$

By running the proposed model Eq. 4 with the initial value $z(0)$ as given by Eq. 26, we can obtain the eigenvector corresponding to the maximum real part eigenvalues, as follows:

$$
z(t)=\left(\begin{array}{c}
1.3910-0.2970 \mathrm{i} \\
-1.0604+0.2264 \mathrm{i} \\
0.1060-0.0226 \mathrm{i} \\
-1.6025+0.3421 \mathrm{i} \\
-0.2276+0.0486 \mathrm{i} \\
3.0329-0.6475 \mathrm{i}
\end{array}\right)=(-3.8586+0.8238 \mathrm{i}) v_{6} .
$$

From above, we can see that the eigenvectors that we got from the complex neural network algorithm are constant multiple of the eigenvector obtained from the direct calculation. One can get the eigenvalue by $\lambda=\frac{\overline{z(t)}^{T} A z(t)}{z(t)^{T} z(t)}=2.9446-0.0000 \mathrm{i}$, which is just the largest real part eigenvalue of $A$.

Fig. 1 illustrates the dynamic behavior of the largest real part of the symmetry matrix A, and Fig. 2 illustrates the dynamic behavior of six components' modulus of the corresponding eigenvector. From these figures, we note that the proposed algorithm also has the fast convergence property, which is just one virtue of parallel computing. In addition, the proposed algorithm is not sensitive to initial value. This virtue makes actual operation a lot more convenient. 


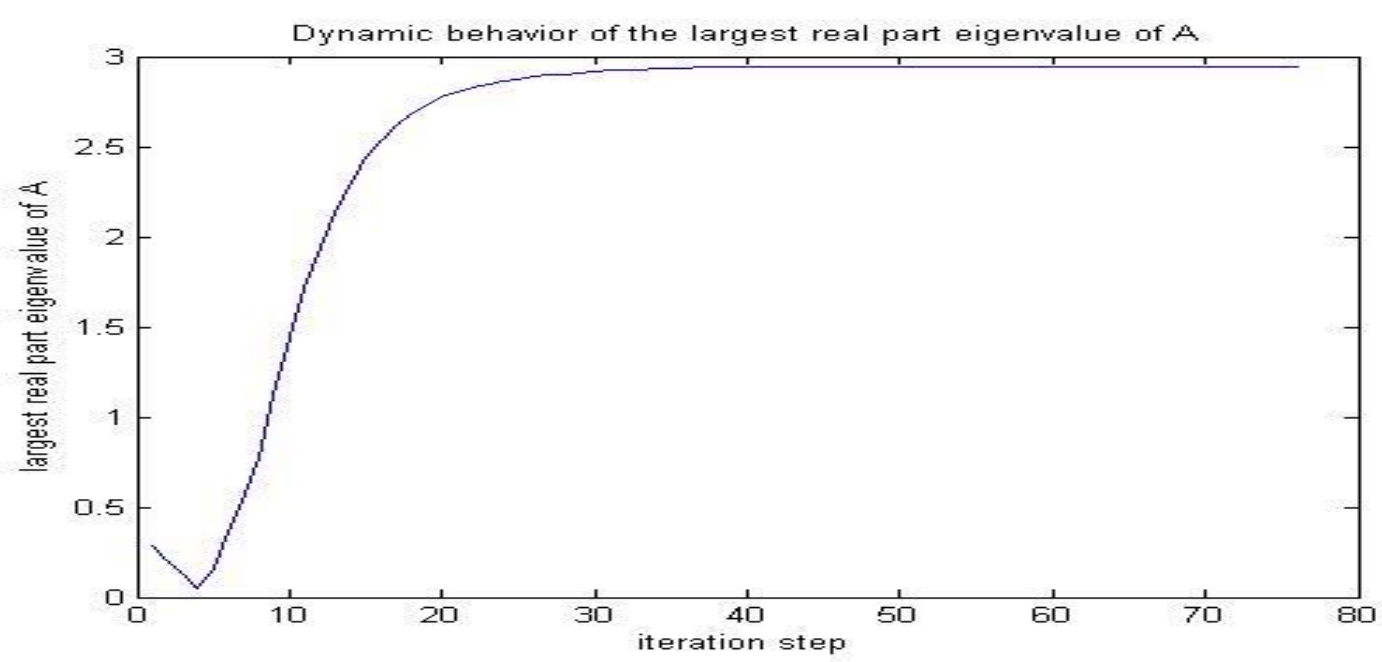

Fig. 1. Dynamic behavior of maximum real part eigenvalue of matrix $A$. It should converge to $\lambda_{6}=2.9446$.

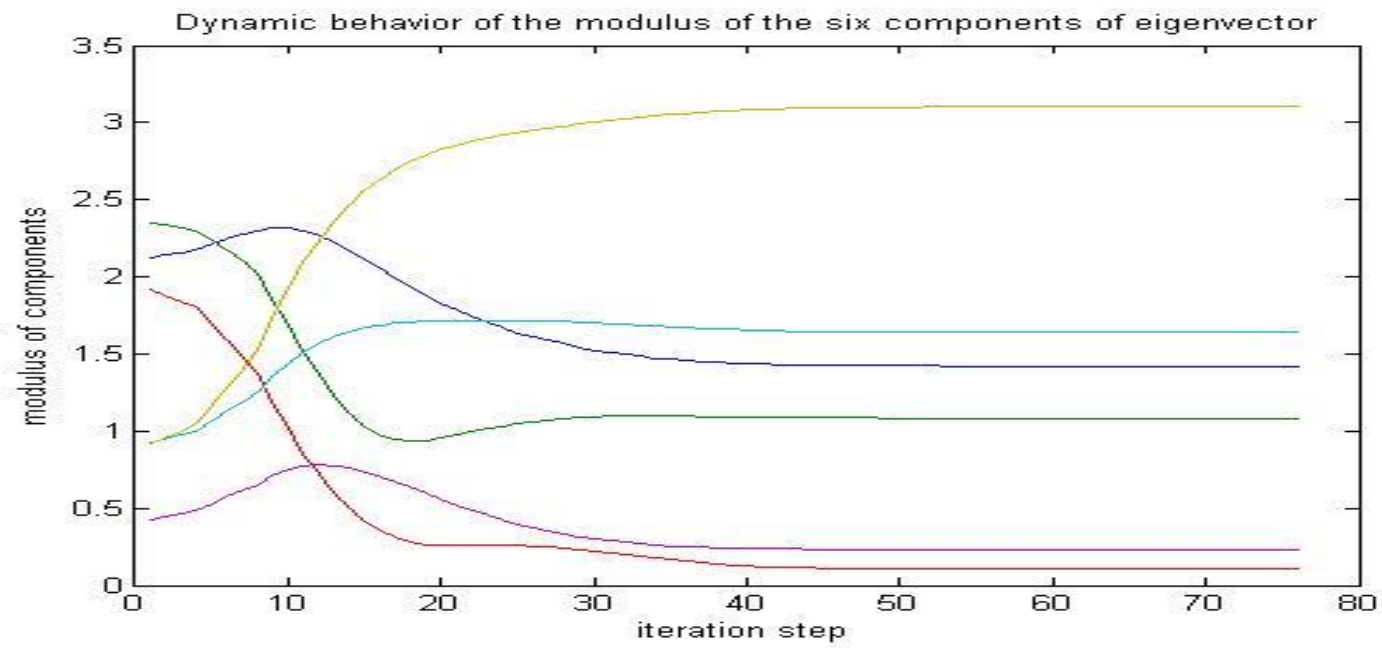

Fig. 2. Dynamic behavior of the modulus of the six components of eigenvector corresponding to the maximum real part eigenvalue. It should converge to the six components' modulus of eigenvector corresponding to eigenvalue $\lambda_{6}=2.9446$.

Example 2. Now we consider a general real matrix $A$. The following is an artificial real matrix:

$$
A=\left(\begin{array}{rrrrrr}
0.0906 & -1.4084 & -0.4040 & 2.1150 & 0.3314 & 0.9130 \\
-0.2233 & 1.3014 & 1.1517 & -0.9942 & -1.2981 & -1.1189 \\
-1.2689 & 0.0854 & 1.1531 & -1.5845 & 1.0717 & 0.2408 \\
0.4824 & 0.4790 & -0.5256 & 0.3109 & 0.4214 & 1.1234 \\
-1.4415 & -0.2628 & 0.4294 & -0.9696 & 1.4224 & -0.6482 \\
-0.3045 & 1.5868 & 0.1266 & -0.5891 & -0.3119 & 0.0874
\end{array}\right)
$$

By directly solving the eigenpairs problems of $A$, we could obtain the eigenvalues of $A$ as follows: $\lambda_{1}=-0.9979, \lambda_{2}=0.6113+1.5384 \mathrm{i}, \lambda_{3}=0.6113-1.5384 \mathrm{i}, \lambda_{4}=2.2769, \lambda_{5}=1.5223$, and $\lambda_{6}=0.3419$. The corresponding eigenvector of the largest real part eigenvalue $\lambda_{4}=2.2769$ is:

$$
v_{4 c}=(-0.1449,0.1376,0.7905,-0.0373,0.5701,0.0944)^{T}
$$

We run the proposed algorithm Eq. 4 with the initial value $z(0)$ also given by Eq. 26 . We can 
obtain the largest real part eigenvalue is $\lambda=\frac{\overline{z(t)}^{T} A z(t)}{z(t)^{T} \overline{z(t)}}=2.2769$, which indeed the directly calculation value, and the corresponding eigenvector as follows:

$$
v_{4}=\left(\begin{array}{c}
0.3863+0.4221 \mathrm{i} \\
-0.3667-0.4007 \mathrm{i} \\
-2.1074-2.3026 \mathrm{i} \\
0.0993+0.1085 \mathrm{i} \\
-1.5198-1.6605 \mathrm{i} \\
-0.2516-0.2749 \mathrm{i}
\end{array}\right)=(-2.6660-2.9129 \mathrm{i}) \mathrm{v}_{4 c},
$$

From which, we can see that the eigenvector obtained from the complex neural network algorithm are also the constant multiples of the eigenvector obtained from the direct calculation. In the following, Fig. 3 illustrates the dynamic behavior of the largest real part of real matrix $A$, and Fig. 4 illustrates the dynamic behavior of six components' modulus of the corresponding eigenvector.

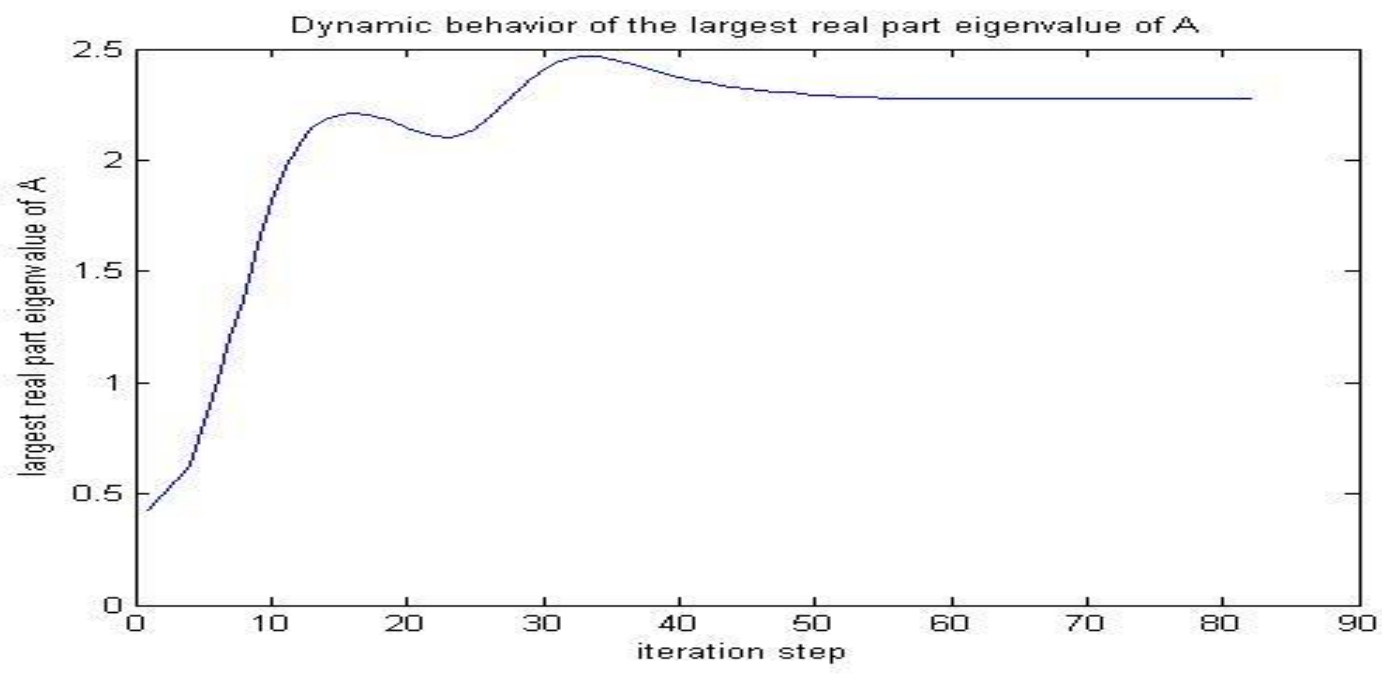

Fig. 3. Dynamic behavior of maximum real part eigenvalue of the real matrix $A$. It should converge to $\lambda_{4}$.

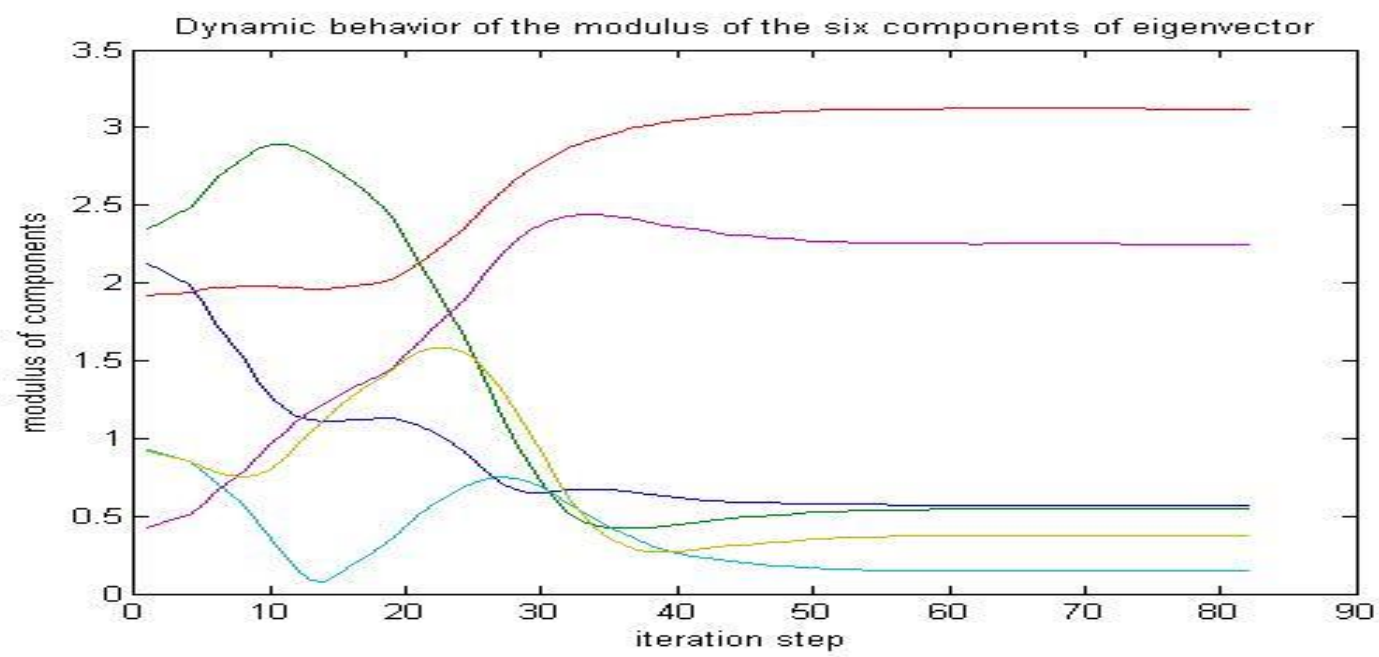

Fig. 4. Dynamic behavior of the modulus of the six components of eigenvector corresponding to the maximum real part eigenvalue. It should converge to the six components' modulus of eigenvector corresponding to eigenvalue $\lambda_{4}$. 


\section{Conclusion}

Based on the classical real domain neural network algorithm, this paper proposed a novel complex neural network to directly compute the largest real part eigenvalue and the corresponding eigenvector of real matrices. Two simulation experiments indicated that the proposed algorithm is effective.

\section{Acknowledgements}

This work was partially supported by the General project of Sichuan Provincial Department of Education (14ZB0331), and The Chengdu Normal University introduces the talented person scientific research start funds subsidization project (YJRC2014-5).

\section{References}

[1] Oja E. A: Journal of Mathematical Biology Vol. 15 (1982), p. 267-273.

[2] Chen Tianping: Chinese Sci Bull Vol. 41 (1995), p. 1344-1344.

[3] Y. Liu, Z.S. You, L.P. Cao: Theoretical Computer Science Vol. 367 (2006), p. 273-285.

[4] Yiguang Liu, Zhisheng You, Liping Cao: Computers and Mathematics with Applications Vol. 53 (2007), p. 41-53.

[5] F.L. Luo, Y.D. Li: Neurocomputing Vol. 7 (1995), p. 145-157.

[6] F.L. Luo, R. Unbehauen, Y.D. Li: Neurocomputing Vol. 8 (1995), p. 213-221.

[7] Y. Zhang, F. Yan, and H.J. Tang: Comput. Math. Appl. Vol. 47(2004), p. 1155-1164.

[8] Y. Liu, Z.S. You, and L.P. Cao: Neurocomputing Vol. 67 (2005), p. 384-397.

[9] Y. Liu, Z.S. You, and L.P. Cao: Theoretical Computer Science Vol. 367 (2006), p. 273-285.

[10] Ying Tang, and J.P. Li: Computers and Mathematics with Applications Vol. 60 (2010), p. $1385-1392$.

[11] T. D. Nguyen, and I. Yamada: IEEE Trans. Signal Process Vol. 61 (6) (2013), p. 1404-1418.

[12] Xiaowei Feng, Xiangyu Kong, Zhansheng Duan, and Hongguang Ma: IEEE TRANSACTIONS ON SIGNAL PROCESSING Vol. 64 (11) ( 2016), p. 2976-2989. 